\title{
CARACTERÍSTICAS CLÍNICO-ONCOLÓGICAS Y SOBREVIDA GLOBAL DE PACIENTES CON TUMOR TESTICULAR DE CÉLULAS GERMINALES REGISTRADOS EN EL HOSPITAL MILITAR CENTRAL “CRL LUIS ARIAS SCHREIBER” EN EL PERIODO DEL 2004 AL 2014
}

\author{
CLINICAL-ONCOLOGICAL CHARACTERISTICS AND GLOBAL SURVIVAL OF PATIENTS WITH TESTICULAR TUMOR OF \\ GERMINAL CELLS REGISTERED IN THE CENTRAL MILITARY HOSPITAL "CRL LUIS ARIAS SCHREIBER"
}

IN THE PERIOD FROM 2004 TO 2014

Robert Malpartida-Palomino ${ }^{1, a}$, Rómulo Cárdenas-Agramonte

\begin{abstract}
RESUMEN
Objetivo: Identificar las características clínico oncológicas y sobrevida global en pacientes con tumor testicular de células germinales seminoma y no seminoma. Métodos: Estudio retrospectivo, en pacientes con tumor testicular de células germinales seminoma y no seminoma, atendidos en el Hospital Central de Militar CRL LUIS ARIAS SCHREIBER, de enero de 2004 a enero de 2014. Variables de estudio: marcadores tumorales, estadio y tratamiento. Finalmente se realizó la supervivencia global. Resultados: Durante el período de estudio de enero del 2004 a enero 2014, se registraron un total de 26 pacientes con diagnostico histopatológico de tumor de células germinales entre seminomas y no seminomas. La presentación histopatológicamente, el tipo más frecuente fue tumor de células germinales fue de tipo no seminoma $(69.23 \%)$, dentro de sus variantes el $33 \%$ fue del tipo tumor mixto, seguido de coriocarcinoma puro con un $28 \%$, carcinoma embrionario puro $17 \%$, saco vitelino $11 \%$ y teratoma puro $11 \%$. Los tumores de células germinales tipo seminoma (30.77\%) dentro de sus variantes el $62.5 \%$ de estos fueron seminomas clásicos y $37.5 \%$ de seminoma multinodular. Durante el período de observación de 179 meses con una media106 meses y una mediana de 101 meses la tasa de supervivencia global a 5 años fue de $92.3 \%$. ( $p<0.05$ ). Conclusión: El presente estudio mostró un buen pronóstico. La supervivencia se atribuye sustancialmente al diagnóstico preciso y al tratamiento eficaz. Con respecto al manejo de esta neoplasia tumor testicular de células germinales seminomas y no seminomas debe ser multidisciplinario.
\end{abstract}

Palabras clave: Tumor testicular;Tumor de células germinales; Seminoma y no seminoma. (fuente: DeCS BIREME)

\begin{abstract}
Objetive: To identify oncological clinical features and global survival in patients with seminoma and nonseminoma germ cell testicular tumor. Methods: Retrospective study in patients with seminoma and nonseminoma germ cell testicular tumors, treated at the Central Military Hospital CRL LUIS ARIAS SCHREIBER, from January 2004 to January 2014. Study variables: tumor markers, stage and treatment. Finally, global survival was realized. Results: During the study period from January 2004 to January 2014, a total of 26 patients were registered with histopathological diagnosis of germ cell tumor between seminomas and nonseminomas. Histopathologically, the most frequent type was germ cell tumor was non-seminoma type (69.23\%), 33\% of its variants were mixed tumor type, followed by pure choriocarcinoma with $28 \%$, pure embryonic carcinoma $17 \%$, $11 \%$ yolk sac and $11 \%$ pure teratoma. The seminoma-type germ cell tumors (30.77\%) within its variants $62.5 \%$ of these were classic seminomas and $37.5 \%$ multinodular seminoma. During the observation period of 179 months with a mean of 106 months and a median of 101 months, the 5 -year overall survival rate was $92.3 \%$. ( $p$ $<0.05$ ). Conclusion: The present study showed a good prognosis. Survival is attributed substantially to accurate diagnosis and effective treatment. Regarding the management of this neoplasm, testicular tumor of germ cell seminomas and non-seminomas must be multidisciplinary.
\end{abstract}

Key words: Testicular tumor; Germ cell tumor; Seminoma and nonseminoma. (source: MeSH NLM)

\footnotetext{
'Servicio de Oncología Médica, Hospital Militar Central, Lima-Perú.

2 Consultor, asesor docente del Hospital Militar Central, Lima-Perú.

aédico especialista en Oncología Médica.
}

Citar como: Robert Malpartida-Palomino, Rómulo Cárdenas-Agramonte. Características clínico-oncológicas y sobrevida global de pacientes con tumor testicular de células germinales registrados en el hospital militar central "CRL Luis Arias Schreiber" en el periodo del 2004 al 2014. [Artículo Original].2018;18(4):16-24. (Octubre 2018). DOI 10.25176/RFMH.v18.n4.1726 


\section{INTRODUCCIÓN}

El cáncer de testículo es la neoplasia maligna sólida más común que afecta a los hombres entre las edades de 15 y 35 años, aunque representa solo el $1 \%$ de todos los cánceres en los hombres'. En el Perú, los jóvenes afectos de cáncer testicular, tienen una historia larga de enfermedad, acudiendo a la consulta cuando la enfermedad ya ha hecho metástasis2, 3. La incidencia de cáncer de testículo en nuestro país es: 1,1 casos nuevos x 100000 varones, la mortalidad: 0,6 100000 varones y la prevalencia a 5 años: $1,9 \%$.

Los tumores de células germinales (TCG) representan el $95 \%$ de los cánceres testiculares. Pueden consistir en un patrón histológico predominante o representar una combinación de múltiples tipos histológicos. Para fines de tratamiento, se reconocen dos categorías amplias de tumores de testículo: seminoma puro (sin elementos no seminomatosos presentes) y todos los demás, que en conjunto se denominan tumores de células germinales no seminomatosas (TCGNS).

El tumor de células germinales se ha convertido en una de las neoplasias sólidas más curables debido a los notables avances en el tratamiento que comenzaron a fines de los años setenta. Antes de ese momento, el tumor de células germinales representaba el 11 por ciento de todas las muertes por cáncer en hombres entre las edades de 25 y 34 años, y la tasa de supervivencia a cinco años era del $64 \%$. Actualmente la tasa de supervivencia a cinco años es superior al 95\%, y aproximadamente 400 muertes por cáncer testicular se ven en los Estados Unidos por año1, 6. Aproximadamente 9300 hombres son diagnosticados con cáncer testicular cada año en los Estados Unidos, pero solo aproximadamente 400 hombres morirán de su enfermedad7. En todo el mundo, hay aproximadamente 72,000 casos y 9000 muertes por año debido al cáncer testicular.

Los TCG de testículo son raros antes de la pubertad8. Los tumores testiculares generalmente se presentan como un nódulo o una inflamación indolora de un testículo, que puede ser un hallazgo incidentalmente por el paciente o por su pareja. Aproximadamente del 30 al $40 \%$ de los pacientes se quejan de un dolor sordo o sensación de pesadez en la parte baja del abdomen, el área perianal o el escroto, mientras que el dolor agudo es el síntoma de presentación en el $10 \%$. En otro $10 \%$, las manifestaciones de presentación del cáncer testicular son atribuibles a la enfermedad metastásica. Los síntomas varían con el sitio de metástasis?.

La evaluación inicial ante la sospecha de un tumor testicular debe incluir un examen físico del testículo complementado con ultrasonido para diferenciar de una lesión benigna (un hidrocele o epididimitis) versus una lesión maligna (tumor) ${ }^{10,11,12}$. La evaluación inicial debe incluir la medición de los marcadores tumorales séricos (la subunidad beta de la gonadotropina coriónica humana (beta-hCG), la alfa-fetoproteína (AFP) y lactato deshidrogenasa (LDH) ${ }^{13}$. Los estudios de imagen deben incluir radiografía de tórax, así como tomografía computarizada (TC) ${ }^{14,15}$ o resonancia magnética (RM) de abdomen y pelvis para buscar evidencia de metástasis en los ganglios linfáticos regionales ${ }^{12}$. Antes del tratamiento definitivo, también debe considerarse la posible necesidad de la crio conservación de espermatozoides ${ }^{16}$.

El diagnóstico de una neoplasia testicular se establece generalmente en la orquiectomía radical, que también sirve como tratamiento inicial para el tumor primario. La terapia óptima requiere estimar la probabilidad de recurrencia para que la terapia pueda limitarse cuando sea apropiado. Los elementos clave en la estratificación del riesgo incluyen la histología (seminoma versus tumor de células germinales no seminomatosas (TCGNS), la presencia o ausencia de metástasis y el grado de elevación en los marcadores tumorales séricos. los pacientes con un seminoma en estadio I, se puede lograr una tasa de curación extremadamente alta con la orquiectomía radical. Por lo general, a esto le sigue la vigilancia activa, pero la quimioterapia con carboplatino de agente único o la radioterapia para los ganglios paraaórticos pueden estar indicados si la vigilancia activa no es apropiada ${ }^{17,18}$. Los pacientes con seminoma en estadio II el tratamiento son con RT o quimioterapia basada en cisplatino, dependiendo de la extensión de la enfermedad retroperitoneal ${ }^{19,20}$. Los tumores no seminomatosos de células germinales en estadio temprano en estadio IA e IB, el enfoque de manejo se basa en la presencia o ausencia de factores de riesgo específicos para la recurrencia: invasión vascular o linfática, carcinoma embrionario que comprende $>40 \%$ del volumen total del tumor, la presencia de elementos del saco vitelino, o marcadores tumorales séricos elevados antes de la orquiectomía que no disminuyen con la vida media esperada. Para los pacientes con factores de riesgo, se recomienda una disección de ganglios linfáticos retroperitoneales (RPLND). Sin embargo, un régimen de dos ciclos a base de cisplatino es una alternativa razonable a la cirugía y con frecuencia son el tratamiento de elección ${ }^{21,22}$. Los hombres con marcadores tumorales persistentemente elevados después de una orquiectomía pero sin otra evidencia de enfermedad (estadio IS) deben tratarse 
con quimioterapia similar a aquellos con enfermedad en estadio III de buen riesgo ${ }^{23,24}$. Para los pacientes con NSGCT en estadio II, el tratamiento depende de la extensión de la enfermedad y el compromiso de los ganglios linfáticos retroperitoneales documentadas patológicamente o por estudios de imagen. Las opciones de tratamiento incluyen RPLND, vigilancia o el uso de quimioterapia basada en cisplatino. Para los hombres con NSGCT patológicos en estadio II después de RPLND, el tratamiento se basa en el grado de compromiso ganglionar ${ }^{25,26}$. En enfermedad avanzada los pacientes con enfermedad avanzada se clasifican en grupos de riesgo bueno, intermedio y bajo que usan el sistema de estratificación de riesgo del Grupo Colaborativo Internacional de Cáncer de Células Germinales (IGCCCG). El sistema IGCCCG tiene en cuenta el sitio del tumor primario, la enfermedad metastásica y los niveles séricos de marcadores tumorales ${ }^{27,28}$. Para los pacientes con enfermedad de buen riesgo, la quimioterapia de combinación basada en cisplatino. Nuestro tratamiento actual en el hospital militar consiste en 2 ciclos de bleomicina, etopósido y cisplatino (BEP). Los pacientes con función pulmonar comprometida o aquellos con riesgo de lesión pulmonar inducida por bleomicina deben tratarse con etopósido y cisplatino (EP). Para los pacientes con enfermedad de riesgo intermedio o riesgo bajo, recomendamos cuatro ciclos de BEP. Un régimen alternativo consiste en etopósido, ifosfamida y cisplatino (VIP) y se prefiere para pacientes con riesgo de lesión pulmonar inducida por bleomicina ${ }^{29,30}$.

El objetivo del presente estudio es describir nuestra experiencia en el manejo de tumores testiculares de células germinales, destacando el patrón clínicopatológico, el resultado del tratamiento y la sobrevida global de los pacientes tratados en el Hospital Militar Central registrados del año 2004 al 2014.

\section{MÉTODOS}

Este trabajo es un estudio retrospectivo que incluyó a todos los pacientes con tumor testicular de células germinales confirmado histopatológicamente en el Hospital Militar Central Crl. Luis Arias Schreiber en el periodo de Enero del 2004 a Enero del 2014. Se evaluaron los signos y síntomas, marcadores tumorales, estadio clínico, tratamiento inicial y datos de supervivencia. El intervalo de supervivencia se calculó a partir de la fecha de la orquiectomía radical. Las tasas de supervivencia se calcularon utilizando el método de Kaplan-Meier y se compararon mediante la prueba log-rank. $\mathrm{P}<0.05$ fue designado como significación estadística.

\section{RESULTADOS}

Durante el período de estudio de enero del 2004 a enero 2014, se registraron un total de 26 pacientes con diagnóstico histopatológico de tumor testicular de células germinales entre seminomas y no seminomas. Donde encontramos que la edad más frecuente de presentación de este tipo de cáncer fue de los 21-30 años de edad con 14 de 26 pacientes, haciendo un porcentaje del 53\%, seguido de 7 pacientes de 10 a 20 años que corresponde a un $26 \%$. (GRAFICO N 1 ) No se encontraron casos de presentación de cáncer en pacientes mayores de 51 años. (GRAFICO N²).

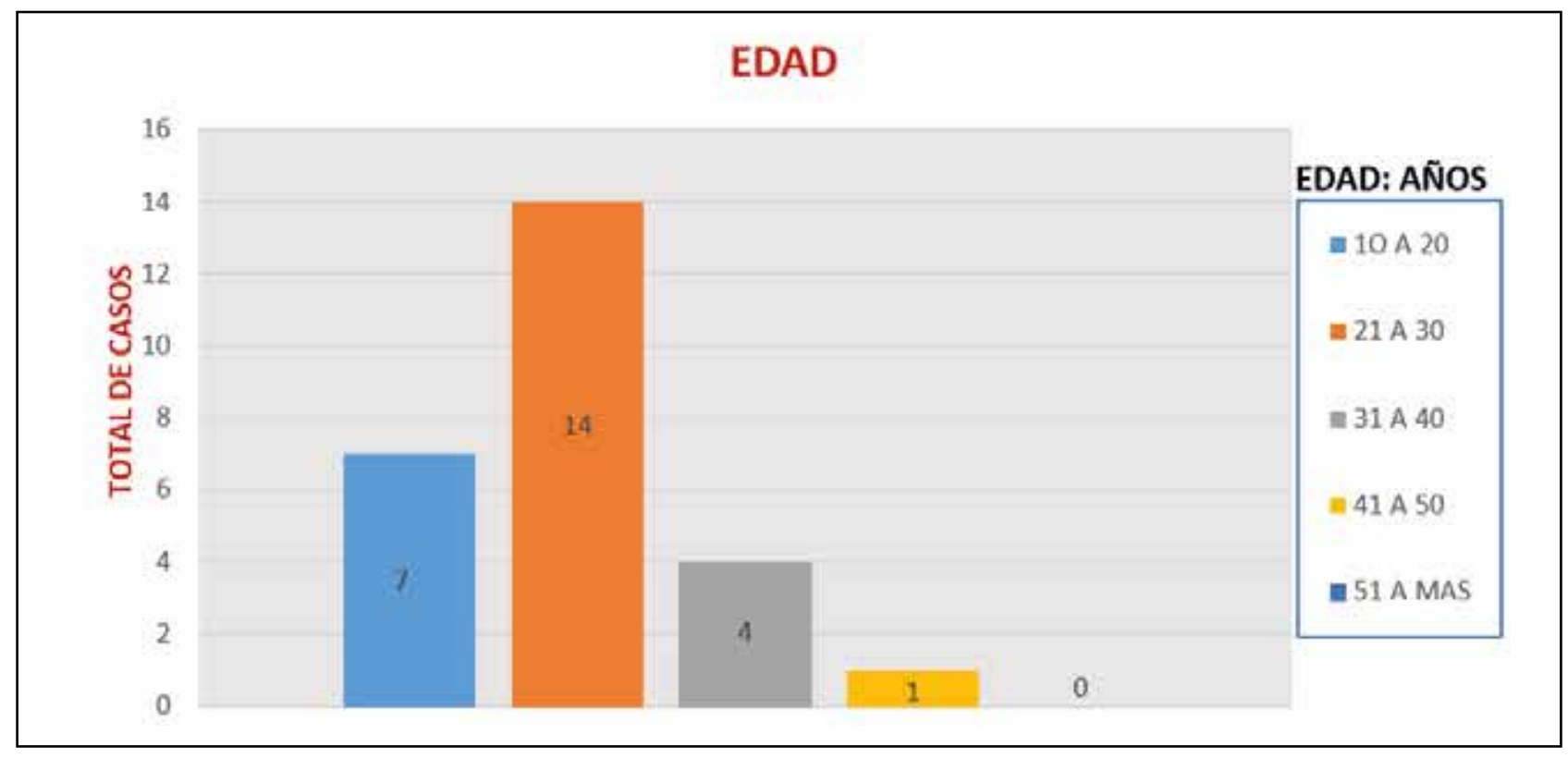

Gráfico 1 . Distribución del total de casos según el grupo de a edad más frecuente de presentación de Cáncer de Células Germinales en el HMC-EP del 2004-2014. 


\section{PORCENTAJE POR EDAD}
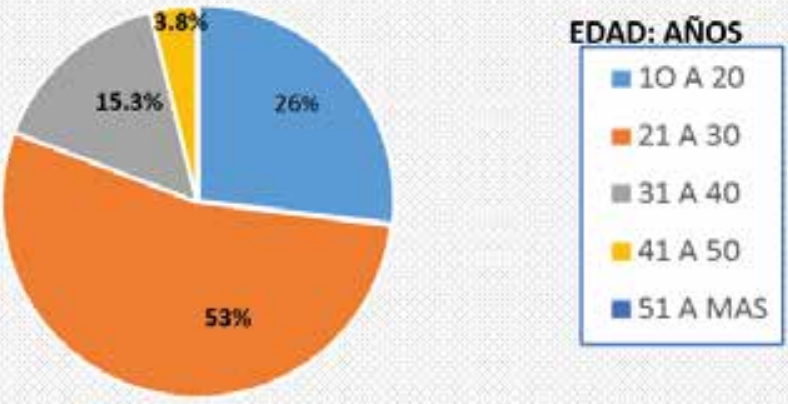

Gráfico 2. Distribución en porcentaje de casos según el grupo de a edad más frecuente de presentación de Cáncer de Células Germinales en el HMC-EP del 2004-2014.

En la población estudiada de 26 pacientes la pacientes. El 30.7\% (8) restante se presentó en el localización más frecuente de presentación es el testículo izquierdo. (GRAFICO N³). testículo derecho en un $69.3 \%$ (18) del total de los

\section{LOCALIZACIÓN}

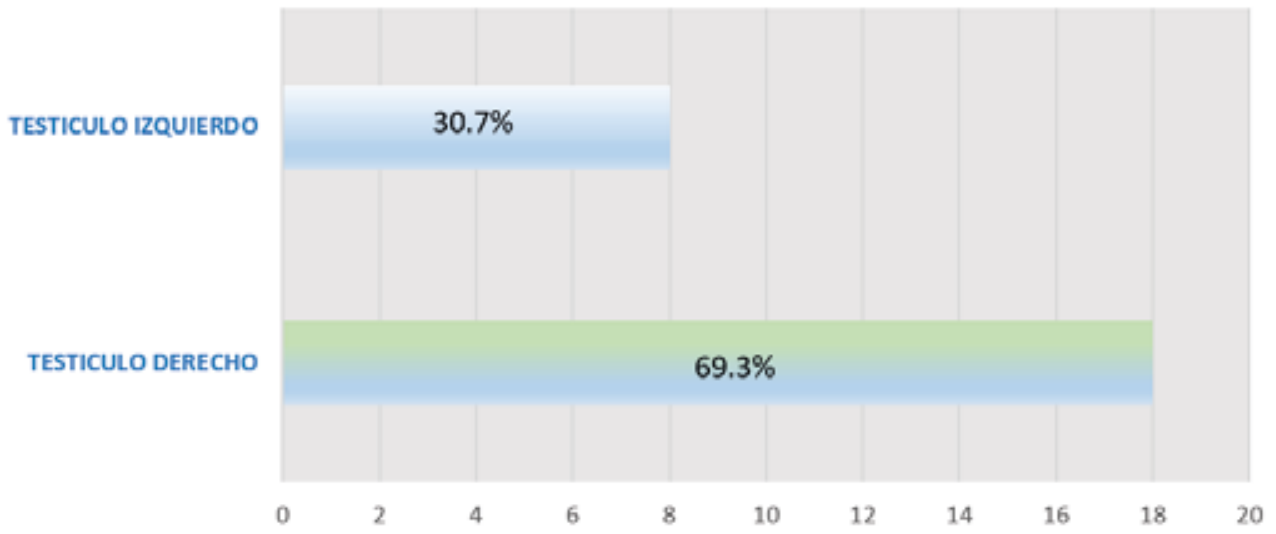

Gráfico 3. Localización testicular más frecuente de la presentación de Cáncer de Células Germinales en el HMC del 2004-2014.

La característica clínica de presentación del tumor de células germinales, fue la aparición o sensación de masa escrotal que se presentó como síntoma único o acompañado de dolor inguinal en algunos casos asociados a fiebre. (GRAFICO Nº4).

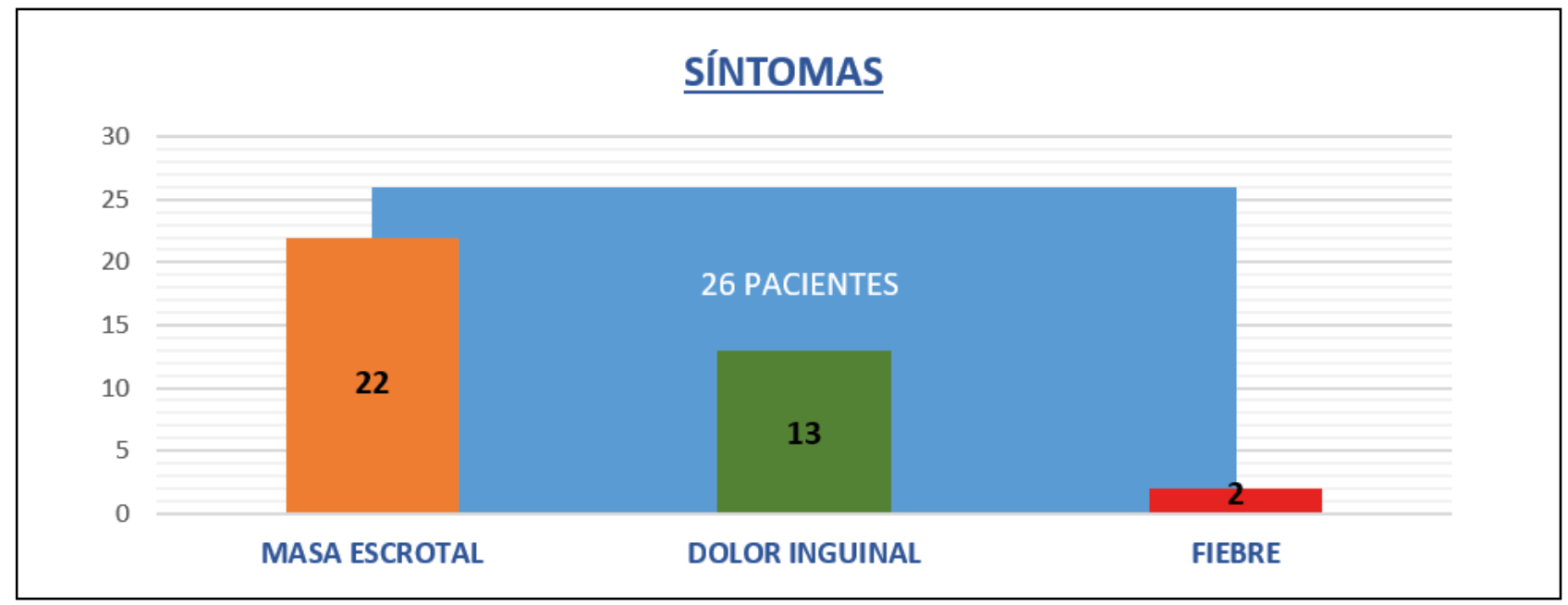

Gráfico 4. Presentación clínica del Cáncer de Células Germinales en el HMC del 2004-2014. 
A los 26 pacientes del presente estudio se les realizó una historia clínica completa y un examen físico, se realizó ecografía escrotal-abdominal, una radiografía de tórax y Tomografía contrastada para detectar la afectación ganglionar retroperitoneal macroscópica.
El procedimiento preoperatorio de marcadores tumorales reveló que el $76.92 \%$ de pacientes curso con valores de LDH (UI): menor a $450 \mathrm{UI} / \mathrm{L}$. y el $23.08 \%$ de pacientes curso con valores elevados de LDH (UI): mayor a $450 \mathrm{UI} / \mathrm{L}$. (GRAFICO N ${ }^{\circ}$ )

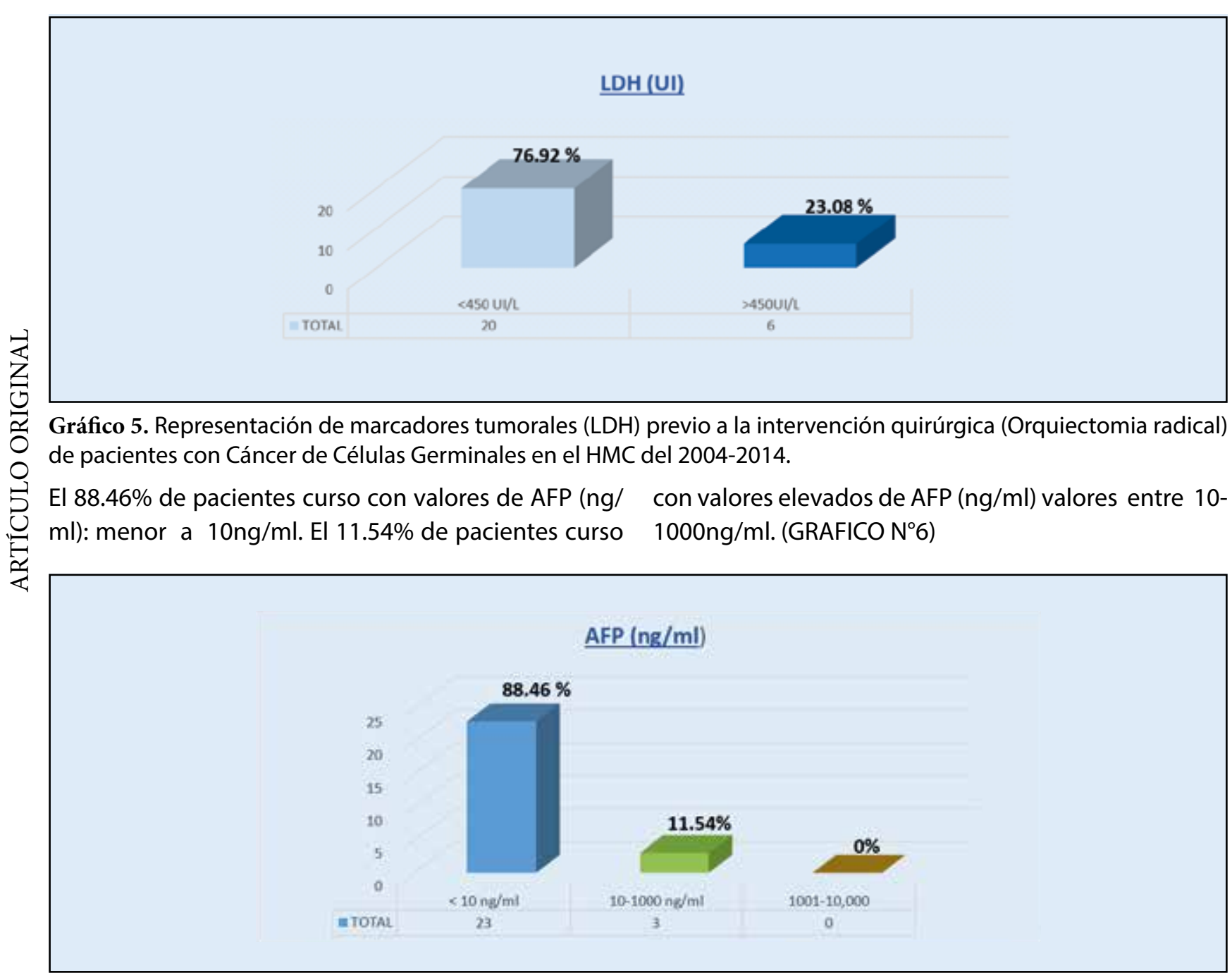

Gráfico 6. Representación de marcadores tumorales (AFP) previo a la intervención quirúrgica (Orquiectomia radical) de pacientes con Cáncer de Células Germinales en el HMC del 2004-2014.

El $80.76 \%$ de pacientes curso con valores de HCG-B curso con valores elevados de HCG-B (ng/ml): entre (ng/ml): menor a $0.1 \mathrm{ng} / \mathrm{ml}$. El $19.24 \%$ de pacientes $0.1-1000 \mathrm{ng} / \mathrm{ml}$. (GRAFICO N).

\section{HCG-B (ng/ml)}

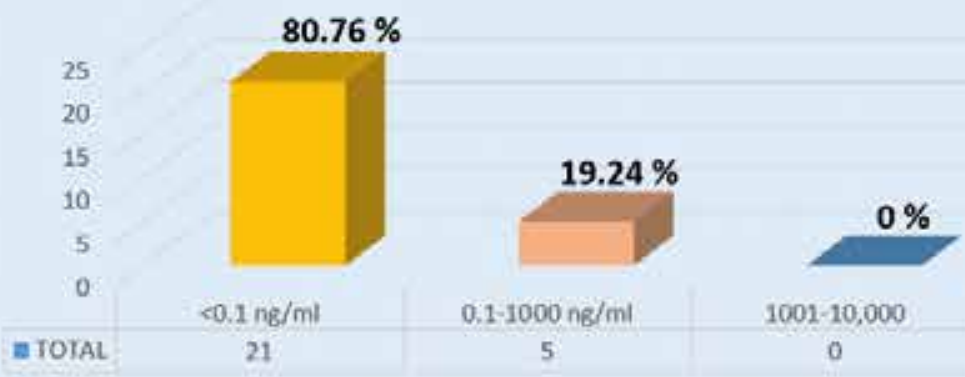

Gráfico 7. Representación de marcadores tumorales (HCG-B) previo a la intervención quirúrgica (Orquiectomia radical) de pacientes con Cáncer de Células Germinales en el HMC del 2004-2014. 
Histopatológicamente, el tipo más frecuente de tumor de células germinales fue de tipo no seminoma (69.23\%), dentro de sus variantes el 33\% fue del tipo tumor mixto, seguido de coriocarcinoma puro con un $28 \%$, carcinoma embrionario puro $17 \%$, saco vitelino
$11 \%$ y teratoma puro $11 \%$. Los tumores de células germinales tipo seminoma (30.77\%) dentro de sus variantes el $62.5 \%$ de estos fueron seminomas clásicos y $37.5 \%$ de seminoma multinodular. (GRAFICO N8)

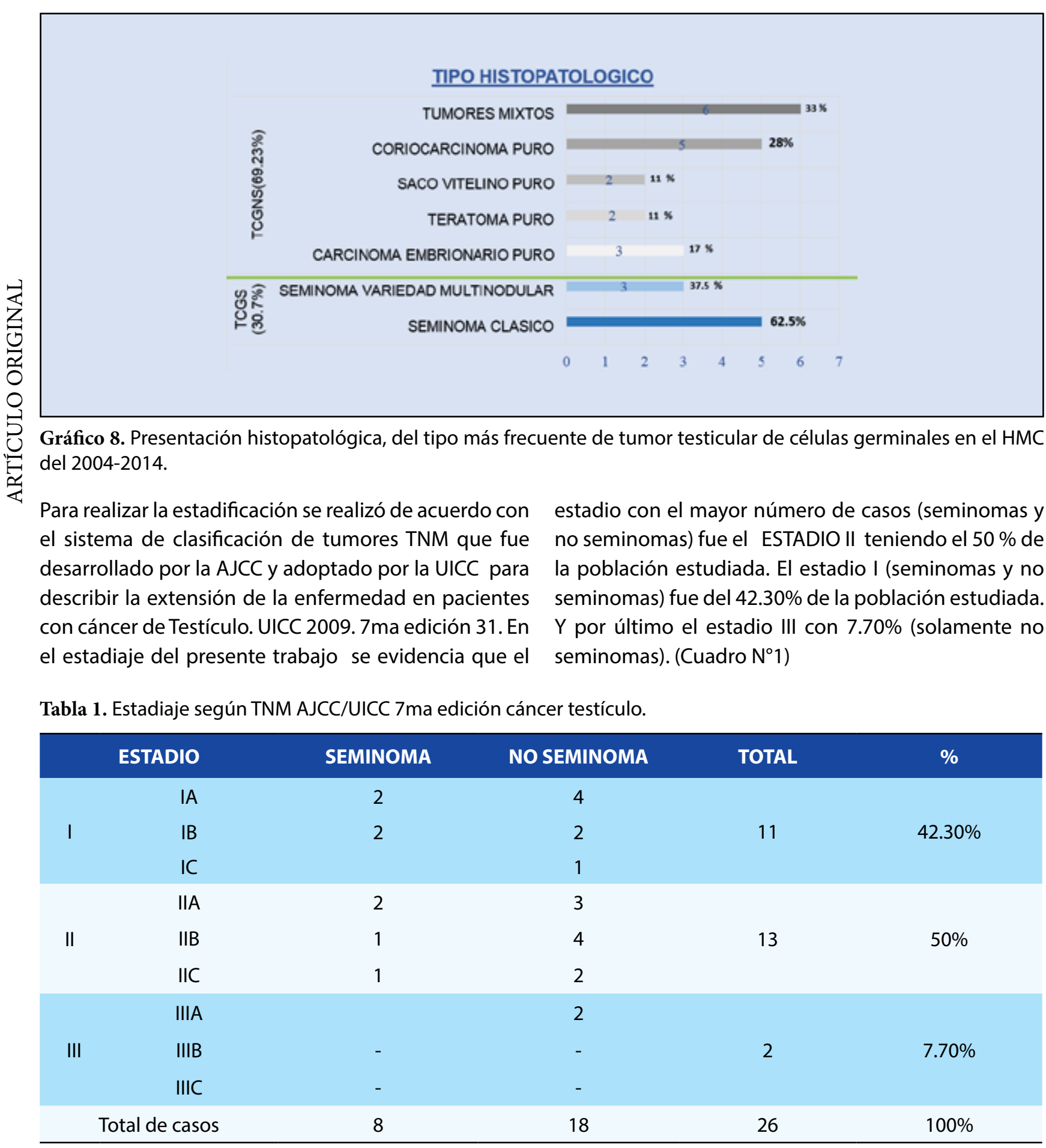

Los pacientes portadores de tumor de células una histopatología de tumor de células germinales germinales (seminomas/no seminomas) de acuerdo al estadiaje obtenido se evidencia que en los estadios IA no seminomas siendo el $61.53 \%$ de la población estudiada. (GRAFICO N9) 


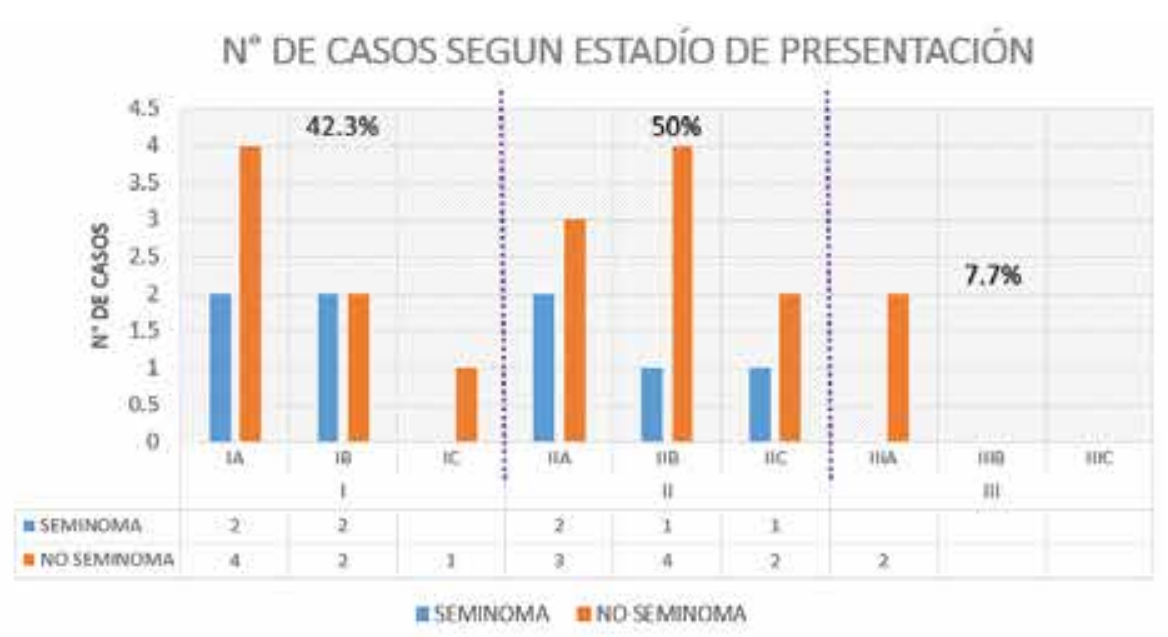

Gráfico 9. Representa el número de casos según Estadío clínico de tumor testicular de células germinales en el HMC del 2004-2014.

Con respecto al tratamiento todos los pacientes fueron orquiectomizados tanto para el diagnóstico y como tratamiento del primario, el siguiente cuadro número 02 se describe que los pacientes con tumor de células germinales seminoma el $62.50 \%$ recibieron quimioterapia adyuvante a base de platinos, y el $37.5 \%$ recibieron radioterapia como tratamiento adyuvante. El cuadro $n 3$ se observa que los pacientes con tumor de células germinales no seminoma en su totalidad recibieron quimioterapia adyuvante donde 12 pacientes recibieron (esquema EP) 5 pacientes recibieron (BEP) y 1 paciente recibió (VIP). 3 de los pacientes hicieron recidiva y fueron sometidos a Resección + Linfadenectomía + Retroperitoneal de los cuales solamente 1 paciente hizo enfermedad viable en el hallazgo anatomopatológico y fue tributario a recibir quimioterapia bajo el esquema VIP.

Tabla 2. Esquemas de tratamiento en tumor de celulas germinales seminoma.

\section{TRATAMIENTO DE TCG SEMINOMA}

\section{TRATAMIENTO POST ORQUIECTOMÍA}

QT ADYUVANTE

RT ADYUVANTE

TOTAL

\section{$\mathbf{N}^{\circ}$}

3

8
$\%$

$62.50 \%$

$37.50 \%$

$100 \%$

Tabla 3. Esquemas de tratamiento en tumor de celulas germinales no seminoma.

TRATAMIENTO DE TCG NO SEMINOMA

TRATAMIENTO POST ORQUIECTOMIA

\section{QX: RESECCIÓN +}

LINFADENECTOMÍA + RETROPERITONEAL

12

5

1
Compromiso retroperitoneal x imágenes3

TOTAL 18 


\section{DATOS DE SUPERIVENCIA}

En el estudio realizado la tasa de supervivencia se calculó utilizando muestra de 26 pacientes que cumplieron con los criterios de estadificación de tumores TNM que fue desarrollado por la AJCC y adoptado por la UICC ${ }^{28,31}$, la histopatología del tumor de células germinales seminoma / no seminoma. Durante el período de observación de 179 meses con una media106 meses y una mediana de 101 meses tasas de supervivencia global a los 5 años fue de 92.3\%. De acuerdo con los criterios de tumor de células germinales tipo seminoma las tasas de supervivencia a 5 años fue de $100 \%$ y en no seminoma fue de $88.9 \%$. De acuerdo con la clasificación por estadiaje en tumor de células germinales en tasas de supervivencia general el estadio I fue del $100 \%$ y en el estadio II, fue de $91.7 \%$ en estadio III, fue del $50 \%$.

Duranteelseguimientosepresentaron 2 Intercurrencias causando como desenlace el fallecimiento de 2 pacientes con tumor de células germinales no seminoma, 1 fallecido por toxicidad pulmonar a Bleomicina y 1 por progresión de enfermedad metastásica y a los 2 y 8 meses respectivamente.

\section{DISCUSIÓN}

El presente trabajo tiene objetivo fue evaluar la distribución de la histología y las etapas clínicas, así como la variación del tratamiento del tumor de células germinales.

En este estudio, la mayoría de los pacientes se encontraban en la segunda y tercera décadas de vida. En la presentación de habitual el cáncer tumor de células germinales seminoma y no seminoma afecta a los hombres jóvenes en la tercera y cuarta décadas de la vida ${ }^{32,33}$.

Los pacientes en este estudio han presentado masa escrotal y dolor se encuentra asociado a una presentación temprana de la enfermedad que aquellos sin dolor ${ }^{34}$. El testículo derecho fue frecuentemente afectado en el presente trabajo ${ }^{35}$.

En este estudio, el diagnóstico de tumores de células germinales seminoma y no seminoma se realizó por motivos clínicos (masa testicular palpable con o sin dolor), ecografía testicular muestras sanguíneas para marcadores tumorales (gonadotropina coriónica $\beta$-humana y a-fetoproteína y lactato deshidrogenasa). Estos marcadores tumorales son útiles en el diagnóstico, la estadificación inicial, la evaluación de la respuesta al tratamiento y la detección temprana de recaídas en los cánceres de células germinales La tomografía computarizada es una herramienta de imagen importante para evaluar la enfermedad metastásica retroperitoneal ${ }^{36}$.

Con respecto a marcadores tumorales se realizó la medición en el laboratorio del hospital militar central EP antes del procedimiento quirúrgico. La orquiectomía radical se realizó en el $100 \%$ de los pacientes como tratamiento para la lesión primaria. La importancia de este procedimiento radica para un diagnóstico histológico correcto. Los hallazgos histopatológicos mostraron una alta prevalencia de tumor de células germinales de tipo seminoma.

Con respecto al tratamiento observamos que la quimioterapia sistémica en tumor de células germinales seminomas, no seminomas se han convertido en una enfermedad tratable. Incluso en pacientes con metástasis, la quimioterapia moderna ofrece una tasa de curación de al menos del $80 \%{ }^{37}$. La terapia BEP y EP se usaron ampliamente tanto para el seminoma como para el no seminoma en más del $90 \%$ de los pacientes en este estudio. Otras combinaciones, como VIP, se utilizaron como quimioterapia de rescate en persistencia de enfermedad en tumor de células germinales no seminoma.

En cuanto a la tasa de supervivencia global a 5 años el estudio actual mostró una supervivencia general a 5 años de más del $92.3 \%$. El presente trabajo es el primer estudio de nuestra institución donde muestra las características y la supervivencia de los pacientes con tumor de células germinales seminomas y no seminomas en hospital militar central EP donde mostró un buen pronóstico, en seminoma y no seminoma. La supervivencia mejorada se atribuyó a un diagnóstico preciso y un tratamiento efectivo.

\section{CONCLUSIÓN}

El presente trabajo sobre tumores testiculares de células germinales seminomas y no seminomas tiene como finalidad dar a conocer que el manejo de los pacientes debe ser multidisciplinario. Por lo descrito anteriormente observamos que los pacientes deben ser tratados con quimioterapia sistémica y radioterapia adyuvante ya que disminuyen la posibilidad de recurrencia en etapas iniciales. Los factores pronósticos son imprescindibles para poder estratificar para que los pacientes puedan obtener mayor beneficio en su tratamiento. 
Contribuciones de autoría: Los autores participaron en la generación, recolección de información, redacción y aprobación final del artículo original.

Financiamiento: Autofinanciado.

Conflicto de interés: Los autores declaran no tener conflicto de interés en la publicación de este artículo.

Recibido: 27 de agosto del 2018

Aprobado: 28 de setiembre del 2018

Correspondencia: Robert Malpartida-Palomino

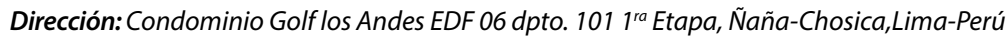

Teléfono: +51981236407

Correo: robertmp7@hotmail.com

\section{REFERENCIAS BIBLIOGRÁFICAS}

1. Siegel RL, Miller KD, Jemal A. Cancer statistics, 2018. CA Cancer J Clin 2018; $68 \cdot 7$.

2. Guías de Práctica Oncológica. Clínica del Cáncer testicular. Instituto Nacional de Enfermedades Neoplásicas (INEN).2013. Lima-Perú

. Leslie Adrianzón S, Guillermo Bendezú A, Isabel Castro C, Salvador Cáceres C, Humberto Riva S, Wilber Tejada. Cancer de testículo. Revista Peruana de Radiologia,1998;2(3):1-3

4. Raúl Alarco, Jorge Baluarte, Iván Aguilar. Reporte de un caso de cáncer de testículo con metástasis inguinal. Carcinos. Revista de Investigación Oncológica, Lima-Perú. 2015.5(1):19.20

5. Registro de Cáncer de Lima Metropolitana. Lima; 2014.

6. Einhorn LH. Treatment of testicular cancer: a new and improved model. J Clin Oncol 1990; 8:1777.

7. Global Burden of Disease Cancer Collaboration, Fitzmaurice C, Allen C, et at. Global, Regional, and National Cancer Incidence, Mortality, Years of Life Lost Years Lived With Disability, and Disability-Adjusted Life-years for 32 Cance Groups, 1990 to 2015: A Systematic Analysis for the Global Burden of Disease Study. JAMA Oncol 2017; 3:524.

8. Walsh TJ, Grady RW, Porter MP, et al. Incidencia de cáncer de células germinales testiculares en niños de EE. UU. experiencia en el programa SEER de 1973 a 2000. Urología 2006; 68: 402.

9. BosI GJ, Motzer RJ. Testicular germ-cell cancer. N Engl J Med 1997; 337:242.

10. Benson CB. The role of ultrasound in diagnosis and staging of testicular cancer. Semin Urol 1988; 6:189.

11. Marth D, Scheidegger J, Studer UE. Ultrasonography of testicular tumors. Urol Int 1990; 45:237

12. Schultz-Lampel D, Bogaert G, Thüroff JW, et al. MRI for evaluation of scrotal pathology. Urol Res 1991; 19:289.

13. Bower M, Rustin GJ. Serum tumor markers and their role in monitoring germ cell cancers of the testis. In: Textbook of Genitourinary Oncology, 2nd Vogelzang NJ, Scardino PT, Shipley,WU, Coffey, DS (Eds), Lippincott, Williams and Wilkins, Philadelphia 2000. p.931.

14. Gels ME, Hoekstra HJ, Sleijfer DT, et al. Detection of recurrence in patients with clinical stage I nonseminomatous testicular germ cell tumors and consequences for further follow-up: a single-center 10-year experience. J Clin Oncol 1995; 13:1188.

15. Socinski MA, Stomper PC. Radiologic evaluation of nonseminomatous germ cell tumor of the testis. Semin Urol 1988; 6:203.

16. Schultz-Lampel D, Bogaert G, Thüroff JW, et al. MRI for evaluation of scrotal pathology. Urol Res 1991; 19:289.

17. Oliver RT, Mason MD, Mead GM, et al. Radiotherapy versus single-dose carboplatin in adjuvant treatment of stage I seminoma: a randomised trial. Lancet 2005; 366:293.

18. Oliver RT, Mead GM, Rustin GJ, et al. Randomized trial of carboplatin versus radiotherapy for stage I seminoma: mature results on relapse and contralatera testis cancer rates in MRC TE19/EORTC 30982 study (ISRCTN27163214). J Clin Oncol 2011; 29:957.

19. Schmoll HJ, Jordan K, Huddart R, et al. Testicular seminoma: ESMO Clinical Practice Guidelines for diagnosis, treatment and follow-up. Ann Oncol 2010; 21 Suppl 5:v140.

20. Wood L, Kollmannsberger $C$, Jewett $M$, et al. Canadian consensus guidelines for the management of testicular germ cell cancer. Can Urol Assoc J 2010; 4:e19.
21. Kollmannsberger $C$, Moore $C$, $C$ hi KN, et al. Non-risk-adapted surveillance for patients with stage I nonseminomatous testicular germ-cell tumors: diminishing treatment-related morbidity while maintaining efficacy. Ann Oncol 2010; 21:1296

22. Sturgeon JF, Moore MJ, Kakiashvili DM, et al. Non-risk-adapted surveillance in clinical stage I nonseminomatous germ cell tumors: the Princess Margaret Hospital's experience. Eur Urol 2011; 59:556.

23. Klepp O, Flodgren $\mathrm{P}$, Maartman-Moe $\mathrm{H}$, et al. Early clinical stages (CS1, CS1Mk+ and CS2A) of non-seminomatous testis cancer. Value of pre- and postorchiectomy serum tumor marker information in prediction of retroperitoneal lymph node metastases. Swedish-Norwegian Testicular Cancer Project (SWENOTECA). Ann Oncol 1990; 1:281.

24. Einhorn LH, Williams SD, Loehrer PJ, et al. Evaluation of optimal duration of chemotherapy in favorable-prognosis disseminated germ cell tumors: a Southeastern Cancer Study Group protocol. J Clin Oncol 1989; 7:387.

25. Beck SD, Foster RS, Bihrle R, et al. Impact of the number of positive lymph nodes on disease-free survival in patients with pathological stage $B 1$ nonseminomatous germ cell tumor. J Urol 2005; 174:143.

26. Hermans BP, Sweeney CJ, Foster RS, et al. Risk of systemic metastases in clinical stage I nonseminoma germ cell testis tumor managed by retroperitoneal lymph node dissection. J Urol 2000; 163:1721.

27. International Germ Cell Consensus Classification: a prognostic factorbased staging system for metastatic germ cell cancers. International Germ Cel Cancer Collaborative Group. J Clin Oncol 1997; 15:594.

28. Testis. In: AJCC Cancer Staging Handbook, 6th, Green FL, Page DL, Fleming ID, et al (Eds), Springer Verlag, New York 2002. p.347.

29. Williams SD, Birch R, Einhorn LH, et al. Treatment of disseminated germ-cell tumors with cisplatin, bleomycin, and either vinblastine or etoposide. $\mathrm{N}$ Engl J Med 1987; 316:1435.

30. Massard C, Plantade A, Gross-Goupil M, et al. Poor prognosis nonseminomatous germ-cell tumours (NSGCTs): should chemotherapy doses be reduced at first cycle to prevent acute respiratory distress syndrome in patients with multiple lung metastases? Ann Oncol 2010; 21:1585.

31. Edge S, Byrd D, Compton C et al, editor: AJCC Cancer Staging Manual, Seventh Edition: New York, NYC: Springer New York Dordrecht Heildeberg London, 2010

32. Richiardi L, Bellocco R, Adami HO, Torrang A, Barlow L, Hakulinen T. Testicular cancer incidence in eight northern European countries: secular and recent trends. Cancer Epidemiol Biomarkers Prev. 2004; 13:2157-2166.

33. Horwich A, Hendry WF. Testicular tumours. Surg. 1988; 53:1266-1270.

34. Cooper DE, L'esperance JO, Christman MS, Auge BK. Testis cancer: a 20 year epidemiological review of the experience at a regional military medical facility. J Urol. 2008; 180:577-581. doi: 10.1016/j.juro.2008.04.032

35. Ugwumba FO, Aghaji AE. Testicular cancer: management challenges in an African developing country. S Afr Med J. 2010; 100:452-455.

36. Ruf CG, Linbecker M, Port M, Riecke A, Schmelz HU, Wagner W, Meineke V Abend $M$. Predicting metastasized seminoma using gene expression. BJU Int 2012; 110:14-20. Doi: 10.1111/j.1464-410X.2011.10778.x.

37. Feldman DR, BosI GJ, Sheinfeld J, Motzer RJ. Tratamiento médico del cáncer testicular avanzado. JAMA 2008; 299: 672-684. 